Article

\title{
Non-Linear Macroeconomic Models of Growth with Memory
}

\author{
Vasily E. Tarasov 1,2 (1) \\ 1 Skobeltsyn Institute of Nuclear Physics, Lomonosov Moscow State University, Moscow 119991, Russia; \\ tarasov@theory.sinp.msu.ru; Tel.: +7-49-5939-5989 \\ 2 Faculty of Information Technologies and Applied Mathematics, Moscow Aviation \\ Institute (National Research University), Moscow 125993, Russia
}

Received: 13 October 2020; Accepted: 13 November 2020; Published: 21 November 2020

\begin{abstract}
In this article, two well-known standard models with continuous time, which are proposed by two Nobel laureates in economics, Robert M. Solow and Robert E. Lucas, are generalized. The continuous time standard models of economic growth do not account for memory effects. Mathematically, this is due to the fact that these models describe equations with derivatives of integer orders. These derivatives are determined by the properties of the function in an infinitely small neighborhood of the considered time. In this article, we proposed two non-linear models of economic growth with memory, for which equations are derived and solutions of these equations are obtained. In the differential equations of these models, instead of the derivative of integer order, fractional derivatives of non-integer order are used, which allow describing long memory with power-law fading. Exact solutions for these non-linear fractional differential equations are obtained. The purpose of this article is to study the influence of memory effects on the rate of economic growth using the proposed simple models with memory as examples. As the methods of this study, exact solutions of fractional differential equations of the proposed models are used. We prove that the effects of memory can significantly (several times) change the growth rate, when other parameters of the model are unchanged.
\end{abstract}

Keywords: fractional differential equation; growth model; fading memory; fractional derivative; solow model

PACS: $45.10 . \mathrm{Hj} ;$ 89.65.Gh

MSC: 26A33; 34A08

\section{Introduction}

The Solow model is a dynamic single-sector model of economic growth (see Solow articles [1,2] and books [3-5]). In this model, the economy is considered without structural subdivisions, i.e., as a one-sector model. It is assumed that the economy produces only universal products, which can be consumed both in the non-production and production consumptions, i.e., can be consumed, $C(t)$, or invested, $I(t)$, (see [4] p. 25). Exports and imports are not taken into account. This model describes the capital accumulation, labor or population growth, and increases in productivity, which is commonly called the technological progress. The Solow model can be used to estimate the separate effects on economic growth of capital, labor, and technological change.

The Solow model is a generalization of the Harrod-Domar model, which includes a productivity growth as new effect. This relatively simple growth model was independently proposed by Robert M. Solow in 1956 [1,2]. In 1987 Solow was awarded the Nobel Memorial Prize in Economic 
Sciences for his contributions to the theory of economic growth [6]. Mathematically, the Solow model is actually represented by the non-linear ordinary differential equation, which describes the evolution of the per capita stock of capital.

In the standard Solow model, the memory effects and memory fading are neglected. From a mathematical point of view, the neglect of memory effects in standard models with continuous time is due to the fact that only equations with derivatives of integer orders are used to describe the economic process. These derivatives are determined by the properties of the function in an infinitely small neighborhood of the considered time. Therefore, standard models of economic growth do not account for memory effects. However, it is obviously strange to assume amnesia in all economic agents. Economic agents can take into account previous changes in economic processes over a certain period of time, and this can influence their decision-making. Therefore, it is important to take memory effects into account when constructing economic models.

The presence of memory in the economic process means that the behavior of the process depends not only on the variables and parameters of this process at the present time, but also on the history of changes in these variables and parameters on a finite time interval [7]. The concept of memory is very important for an adequate description of real economic processes (for example, see [8-20]). For the first time, the importance of long-range time dependence in economic data was recognized by Clive W.J. Granger in his article [9] in 1966 (see also [8,10,11,14]). Granger showed that a number of spectral densities, which are estimated from economic time series, have a similar form. We can state that the phenomenon of long memory in modern economics was discovered by Granger. Then, to describe economic processes with memory Granger and Joyeux [11] in 1980 proposed the fractional ARIMA models, which are also called ARFIMA $(p, d, q)$. The fractional ARIMA $(p, d, q)$ models are generalization of ARIMA $(\mathrm{p}, \mathrm{d}, \mathrm{q})$ model from a positive integer order $d$ to non-integer (positive and negative) orders $d[14,15]$. To generalize ARIMA models, Granger and Joyeux [11] proposed the so-called fractional differencing and integrating for discrete time case (see books [14-19] and reviews [20-24]). Note that Granger received the Nobel Memorial Prize in Economic Sciences in 2003 "for methods of analyzing economic time series with common trends (cointegration)" [13]. The fractional difference operators of Granger and Joyeux were proposed and then began to be used in economics up to the present time without any connection with the fractional calculus and the well-known fractional differences of non-integer orders. In fact, these fractional differencing and integrating are the well-known Grunwald-Letnikov fractional differences, which were suggested in 1867 and 1868 in works [25,26]. Then, the Grunwald-Letnikov fractional differences are actively used in the fractional calculus [27-32] and began to apply in physics and other sciences. We should also note that in the continuous limit the Grunwald-Letnikov fractional differences of positive orders can give the Grunwald-Letnikov, Marchaud, and Liouville fractional derivatives [27].

The approach to describing economic processes with memory, based on discrete operators of Granger and Joyeux, is the most widespread among economists [21-24]. We should note that this approach is really based on the Grunwald-Letnikov fractional differences and it is restricted by only one type of fractional finite differences. Unfortunately, this approach is used without an explicit connection with the modern mathematics and the development of fractional calculus in the last two hundred years. It should be emphasized that the Granger-Joyeux approach to economics with memory is restricted by models with discrete time and application of the Grunwald-Letnikov fractional differences. Obviously, the restriction of mathematical tools to only to the discrete Grunwald-Letnikov operators significantly reduces the possibilities to describe processes with memory and non-locality in time.

In recent years, various linear economic models with fading memory have been investigated. In the framework of such models, the presence of various nontrivial effects was proved due to taking into account fading memory with the remaining other parameters of the models unchanged. For example, the following models have been proposed: The Harrod-Domar model with memory [7,33-35], the Evans model with memory $[33,36]$, and the dynamic Leontief (intersectoral) model with memory $[33,37,38]$. 
A more detailed overview of economic models with memory is presented in work [39] (see also book [40] pp. 5-32 and references therein).

However, non-linear economic models with fading memory and exact solutions of fractional differential equations of such models have not been investigated. This article proposes two non-linear models of economic growth with memory. The suggested models are generalizations of two well-known models, which are proposed by two Nobel laureates in economics, Robert M. Solow and Robert E. Lucas in works [1,41], respectively.

The first proposed model is a generalization of the model of long-run growth, which is considered Robert M. Solow in paper "A contribution to the theory of economic growth" [1]. In the standard model, the capital depreciation is neglected. In the proposed generalization, we take into account the power-law memory. Moreover, we assume the power-law form of the dynamics of the labor and knowledge. The exact analytical solution of the non-linear fractional differential equation, which describes the proposed model, is suggested.

The second proposed model is the standard growth model for closed economy without capital depreciation, which is considered by Robert E. Lucas in paper "Making a Miracle" [41], (see also [42-45]). In the generalized model, we take into account the memory with power-law fading [46]. The suggested model with memory is described by the non-linear fractional differential equation. The explicit expression of analytical solution of this non-linear equation is obtained.

The aim of the work is not to search for memory effects in economics, but to provide mathematical proof of the possibility of the existence of (at least) acceleration in growth rates, when the parameter of memory fading changes, while other parameters of the process remain unchanged.

The main hypothesis used in this article is the assumption of the power-law character of memory fading, which is described by a single fading parameter. The assumption allows us to use fractional calculus and the fractional differential equations [27-32], where the non-integer order of the derivatives and integrals is described by this fading parameter.

We should note that the power-law form of memory fading is distinguished by the fact that this form can be considered as a first approximation for wide class of memory functions. In paper [47], using the generalized Taylor series in the Trujillo-Rivero-Bonilla form, we proved that the memory function for a wide class of functions can be represented through the sum of one or several power-law memory functions. As a result, the integr-differential equations of economic models with general memory functions approximately can be written [47] through the Riemann-Liouville fractional integrals and the Caputo fractional derivatives of non-integer orders [29-32,48].

In this article, simple non-linear economic models with memory are investigated by using the obtained exact solutions of the fractional differential equations of the proposed models. The main aim of this article is to study the possible influence of memory effects on the rate of economic growth using the proposed simple models. As the methods of this study, we use exact solutions of equations, which describe economic growth with power-law memory. Expressions of the warranted rates of growth with memory are suggested and compared with the warranted rates of standard models. We prove that the effects of memory can significantly (several times) change the growth rate, when other parameters of the model are unchanged.

Note that non-linear growth models with continuous time, which take into account memory effects, have not previously been proposed in the modern literature. The exact solutions of the equations of these models and expressions for growth rates which take into account the influence of memory effects have not been proposed before this article.

The article is organized as follows. In Section 2, an economic model with memory is proposed, which is a generalization of the well-known Solow model, considered by Robert M. Solow in paper "A contribution to the theory of economic growth" [1]. In Section 3, we proposed an economic model with memory, which is a generalization of the well-known Solow model, considered by Robert E. Lucas in paper "Making a Miracle" [41]. In each section, the standard models of economic growth without memory are first considered, then the equations of the generalized models with memory are derived, 
solutions of these equations are obtained, expressions of the warranted rate of growth with memory are derived, and then compared with the growth rate without memory. In the last paragraphs of Sections 2 and 3, three principles are formulated that make it possible to qualitatively describe the changes arising in the behavior of the economic process, when taking into account the effects of power memory. Finally, brief conclusions of the article are drawn.

\section{Solow Model of Long-Run Growth with Memory}

In this section, we consider a generalization of the model of long-run growth, which is considered in Solow's paper "A contribution to the theory of economic growth" [1]. In this model, the capital depreciation is neglected. In the proposed generalization, we take into account the power-law memory. Moreover, we assume the power-law form of the dynamics of the labor and knowledge. The exact analytical solution of the non-linear fractional differential equation, which describes the proposed model, is suggested.

\subsection{Long-Run Growth without Memory}

Let us describe the standard economic model of growth, which is considered in Solow's paper [1] pp. 66-67. The Solow model uses four variables: Output $Y(t)$, capital $K(t)$, labor $L(t)$, and knowledge $A(t)$. At any time, the economy has some amounts of capital, labor, and knowledge, which are combined to produce output. The production function is considered in the following form:

$$
Y(t)=F(K(t), A(t) L(t)) .
$$

The Solow model uses assumptions about how the stocks of labor $L(t)$, knowledge $A(t)$, and capital $K(t)$ change over time. The initial levels of capital, labor, and knowledge are taken as given, and are assumed to be strictly positive. In the standard model, the labor and knowledge growth are described [3] p. 13, by the following equations:

$$
\begin{aligned}
& L^{(1)}(t)=\rho L(t), \\
& A^{(1)}(t)=g A(t),
\end{aligned}
$$

where $\rho$ and $g$ are exogenous parameters and $f^{(1)}(t)$ denotes a first derivative with respect to time. The solutions of Equations (2) and (3) can be represented in the following form:

$$
L(t)=L\left(t_{0}\right) \exp \left\{\rho\left(t-t_{0}\right)\right\}, A(t)=A\left(t_{0}\right) \exp \left\{g\left(t-t_{0}\right)\right\}
$$

Let us consider a power-law generalization of Equations (2) and (3) in the following form:

$$
\begin{aligned}
& L^{(1)}(t)=\rho L^{q}(t), \\
& A^{(1)}(t)=g A^{p}(t),
\end{aligned}
$$

where $q$ and $p$ are exogenous parameters. For $q=1$ and $p=1$, Equations (5) and (6) give the standard Equations (2) and (3).

Let us obtain solutions of Equations (5) and (6) for the case $q \neq 1$ and $p \neq 1$.

Equation (5) can be written as:

$$
L^{-q}(t) L^{(1)}(t)=\rho .
$$

Using the standard chain rule, we get:

$$
\frac{d}{d t}\left(L^{1-q}(t)\right)=\rho(1-q)
$$


The solution of Equation (8) has the following form:

$$
L^{1-q}(t)=\rho(1-q) t+c,
$$

where $c=L^{1-q}(0)$. For simplicity, we will assume that $L(0)=0$. Then, $c=0$ and Equation (9) is given as:

$$
L(t)=(\rho(1-q) t)^{1 /(1-q)},
$$

where $t>0$ and $\rho(1-q)>0$. For $t=t_{0}>0$, Equation (10) has the following form:

$$
L\left(t_{0}\right)=\left(\rho(1-q) t_{0}\right)^{1 /(1-q)} .
$$

Using expression (11), we can represent solution (10) by the following expression:

$$
L(t)=L\left(t_{0}\right)\left(\frac{t}{t_{0}}\right)^{1 /(1-q)} .
$$

Analogously, we get:

$$
A(t)=(g(1-p) t)^{1 /(1-p)},
$$

where we assume that $A(0)=0$. Then, we represent solution (13) in the following form:

$$
A(t)=A\left(t_{0}\right)\left(\frac{t}{t_{0}}\right)^{1 /(1-p)} .
$$

Output $Y(t)$ is divided between the consumption $C(t)$ and investment $I(t)$. In the standard model, the fraction $s \in[0,1]$ of the output devoted to investment is exogenous and constant such that:

$$
I(t)=s Y(t) .
$$

Equation (15) describes the economic multiplier without memory and lag.

It is known that depreciation (and disposal of fixed assets) can be described as a truncated exponential distribution of the time lag (up to a numerical factor), which can be represented as a differential equation of the first order ([49] p. 20, [50] p. 86, [51] pp. 252-253 (see also [52] pp. 25-27)).

In a general case, the depreciation can be described by the following equation:

$$
K(t)=\int_{0}^{t} D(t-\tau) I(\tau) d \tau,
$$

where $D(t-\tau)$ is the kernel of the integral operator that describes the depreciation. The kernel $D(t-\tau)$ characterizes the share of fixed assets put into the operation at the time and continuing to operate at time $t>\tau$.

In economics, depreciation is usually described by the kernel in the following exponential form:

$$
D(t-\tau)=e^{-\delta(t-\tau)}
$$

Therefore, the standard form of depreciation and the exponential distributed lag up to a numerical factor can be considered as a truncated mathematical expectation value with an exponential distributed delay time in the following form:

$$
K(t)=\int_{0}^{t} e^{-\delta(t-\tau)} I(\tau) d \tau
$$


Equation (18) can be represented in the form of the ordinary differential equation of the first order. Let us consider the derivative of the first order for Equation (18). Then, we get:

$$
\begin{gathered}
\frac{d K(t)}{d t}=\frac{d}{d t}\left(e^{-\delta t} \int_{0}^{t} e^{\delta \tau} I(\tau) d \tau\right)= \\
\frac{d}{d t}\left(e^{-\delta t}\right) \int_{0}^{t} e^{\delta \tau} I(\tau) d \tau+e^{-\delta t} \frac{d}{d t}\left(\int_{0}^{t} e^{\delta \tau} I(\tau) d \tau\right)= \\
-\delta e^{-\delta t} \int_{0}^{t} e^{\delta \tau} I(\tau) d \tau+e^{-\delta t} e^{\delta t} I(t)=-\delta K(t)+I(t) .
\end{gathered}
$$

As a result, Equation (18) gives:

$$
\frac{d K(t)}{d t}=-\delta K(t)+I(t) .
$$

Substituting expression (20) into Equation (21), that is, taking into account that the capital depreciates at a rate of $\delta \in[0,1]$, we obtain for the standard model the following differential equation:

$$
K^{(1)}(t)=s Y(t)-\delta K(t) .
$$

Let us consider the model without capital depreciation, i.e., $\delta=0$. Then, Equation (21) takes the following form:

$$
K^{(1)}(t)=s Y(t) .
$$

The substitution of (1) into Equation (22) gives:

$$
K^{(1)}(t)=s F(K(t), A(t) L(t)),
$$

where $L(t)$ and $A(t)$ are described by Equations (12) and (14). The substitution of (12) and (14) into Equation (23) gives:

$$
K^{(1)}(t)=s F\left(K(t), A\left(t_{0}\right) L\left(t_{0}\right)\left(t / t_{0}\right)^{1 /(1-p)+1 /(1-q)}\right),
$$

where $q \neq 1$ and $p \neq 1$.

The solution of Equation (24), which describes a model without memory, will be obtained as a special case of the generalized model, when the memory fading parameter is equal to one which means the absence of memory $[7,33]$.

\subsection{Long-Run Growth with Power-Law Memory}

We should note that Equation (22) cannot take into account the memory, since the derivative $K^{(1)}(t)$ is determined by the behavior of $Y(\tau)$ only at the same time instant $\tau=t$, and does not take into account the history of changes of $Y(\tau)$ in the past, when $\tau \in(0, t)$.

To take into account the changes of $Y(\tau)$ in the past, instead of Equation (15), we can consider the equation of the multiplier with memory in the following form:

$$
I(t)=\int_{0}^{t} s(t-\tau) Y(\tau) d \tau,
$$

where the function $s(t-\tau)$ allows us to take into account the changes of output $Y(\tau)$ in the past $\tau \in(0, t)$. The function $s(t-\tau)$ is interpreted as a memory function. In order to have standard dimensions of economic quantities, the time variable $t$ is considered as a dimensionless parameter, i.e., we change the variable $t_{\text {old }} \rightarrow t_{\text {new }}=t_{\text {old }} / t_{c}$, where $t_{c}$ is a characteristic time of processes. 
The sequential action of the memory effects, which are described by Equation (25) and the depreciation, which is described by Equation (17) can be represented in the following form:

$$
K(t)=\int_{0}^{t} M(t-\tau) Y(\tau) d \tau
$$

where we use the associativity of the Laplace convolution * and the kernel:

$$
M(t)=(D * s)(t)=\int_{0}^{t} D(t-\tau) s(\tau) d \tau
$$

This representation allows us to interpret Equation (26) as an equation describing some generalized form of memory and/or depreciation.

If the integral operator in (26) is a fractional integral operator, then for such an operator a left inverse operator, which is a fractional derivative must exist [48]. We should emphasize that the main property of any generalized (fractional) derivative is to be a left-inverse operator to the corresponding generalized (fractional) integral operator. This requirement is important for a self-consistent mathematical theory of the fractional operators to have a general fractional calculus of these operators.

For example, if the integral operator in (26) is the Riemann-Liouville fractional integral $I_{R L, 0+}^{\alpha}$ of the order $\alpha>0$, then Equation (26) can be rewritten in the following form:

$$
K(t)=s\left(I_{R L, 0+}^{\alpha} Y\right)(t) .
$$

Considering the action of the Caputo fractional derivative $D_{C, 0+}^{\alpha}$ and taking into account that this derivative is the left inverse to the integral operator $I_{R L, 0+}^{\alpha}$, we obtain the following equation:

$$
\left(D_{C, 0+}^{\alpha} K\right)(t)=s Y(t) .
$$

The parameter $\alpha>0$ is the order of the fractional derivative or integral. This parameter is interpreted as a memory fading parameter. For integer values of $\alpha$, these fractional operators take the form of standard integrals and derivatives of integer orders (for example, $\left(D_{C, 0+}^{1} K\right)(t)=K^{(1)}(t)$ ).

The path described briefly opens up broad prospects for accounting for sequential and simultaneous actions of two and three effects, namely, fading memory, depreciation, and distributed lag.

To simplify the description, this article will consider a more simple way of describing the effect of power memory based on exact analytical expressions for solutions. The substitution of expression (25) into Equation (21) with $\delta=0$ gives:

$$
K^{(1)}(t)=\int_{0}^{t} s(t, \tau) Y(\tau) d \tau
$$

Let us consider the power-law memory. In this case, the memory function can be described by the following equation:

$$
s(t, \tau)=\frac{s}{\Gamma(\mu)}(t-\tau)^{\mu-1}
$$

where $s$ is the savings parameter, and $\Gamma(\alpha)$ is the gamma function. For function (31), Equation (30) can be written in the following form:

$$
K^{(1)}(t)=s\left(I_{R L ; 0+}^{\mu} Y\right)(t)
$$

where $I_{R L ; 0+}^{\mu}$ is the Riemann-Liouville fractional integral [29] of the order $\mu>0$. Note that Equation (32) with the Riemann-Liouville fractional integral can be considered $[33,47]$ as an approximation of Equation (30) with generalized memory functions $s(t, \tau)$. 
Using the property that the Caputo fractional derivative is the left inverse operator for the Riemann-Liouville fractional integral (see Lemma 2.21 of [29] p. 95), the action of the Caputo derivative on Equation (32) gives:

$$
\left(D_{C ; 0+}^{\mu+1} K\right)(t)=s Y(t)
$$

where $D_{C ; 0+}^{\mu}$ is the Caputo fractional derivative [29] of the order $\mu>0$.

As a result, to take into account the power-law memory, we can use the following equation:

$$
\left(D_{C ; 0+}^{\alpha} K\right)(t)=s Y(t)
$$

where $D_{C ; 0+}^{\alpha}$ is the Caputo fractional derivative of the order $\alpha=\mu+1>1$ with $\mu>0$, i.e., $\alpha>1$. In the general case, we can consider Equation (34) for the order $\alpha>0$ including $\alpha \in(0,1)$.

Note that Equation (34) coincides with Equation (29), which is based on the approach described at the beginning of this subsection.

Let us consider the specific example of a production function. We will use the Cobb-Douglas function. In this case, Equation (1) takes the following form:

$$
Y(t)=K^{a}(t)(A(t) L(t))^{b},
$$

where we can consider $b=1-a$. This production function is easy to analyze, and it appears to be a good first approximation to actual production functions. Therefore, the Cobb-Douglas function is useful for modeling.

The substitution of expression (35) into Equation (34) gives:

$$
\left(D_{C ; 0+}^{\alpha} K\right)(t)=s(A(t) L(t))^{b} K^{a}(t) .
$$

Then, substituting expressions (12) and (14) into Equation (36), we obtain the following equation:

$$
\left(D_{C ; 0+}^{\alpha} K\right)(t)=\lambda\left(t_{0}\right) t^{\beta} K^{a}(t),
$$

where

$$
\begin{aligned}
\lambda\left(t_{0}\right) & =s A^{b}\left(t_{0}\right) L^{b}\left(t_{0}\right) t_{0}^{-\beta}, \\
\beta & =\frac{b}{1-p}+\frac{b}{1-q} .
\end{aligned}
$$

Equation (37) is the non-linear fractional differential equation that describes the generalization of the Solow model without capital depreciation, where we take into account the power-law fading memory.

Using equations 3.5.47 and 3.5.48 of Section 3.5.3 in [29], p. 209, Equation (37) with $\lambda\left(t_{0}\right) \neq 0$, $a, q, p \neq 1$, and $\alpha>0$ has the following solution:

$$
K(t)=\left(\frac{\Gamma(\alpha-\gamma(\alpha)+1)}{\lambda\left(t_{0}\right) \Gamma(1-\gamma(\alpha))}\right)^{1 /(a-1)} t^{\alpha-\gamma(\alpha)}
$$

where

$$
\gamma(\alpha)=\frac{\beta+a \alpha}{a-1}, \alpha-\gamma(\alpha)=\frac{\alpha+\beta}{1-a},
$$

and $\lambda\left(t_{0}\right)$ is defined by (38), $\beta$ is defined by Equation (39).

For $t=t_{0}$, Equation (40) has the following form:

$$
K\left(t_{0}\right)=\left(\frac{\Gamma(\alpha-\gamma(\alpha)+1)}{\lambda\left(t_{0}\right) \Gamma(1-\gamma(\alpha))}\right)^{1 /(a-1)} t_{0}^{\alpha-\gamma(\alpha)} .
$$


Using Equation (42), solution (40) can be written as:

$$
K(t)=K\left(t_{0}\right)\left(\frac{t}{t_{0}}\right)^{\alpha-\gamma(\alpha)} .
$$

The Caputo fractional derivative of the power-law function is given (see Example 3.1 of book [30] p. 49) by the following equation:

$$
\left(D_{C ; t_{0}+}^{\alpha} \tau^{\delta}\right)(t)=\frac{\Gamma(\delta+1)}{\Gamma(\delta-\alpha+1)} t^{\delta-\alpha},
$$

if $\delta>n-1$ and

$$
\left(D_{C ; t_{0}+}^{\alpha} \tau^{\delta}\right)(t)=0,
$$

if $\delta=0,1, \ldots n-1$. In all the remaining cases $(\delta<n-1$ such that $\delta \neq 0,1, \ldots, n-1)$, the integral in the definition of the Caputo fractional derivative is improper and divergent.

Remark 1. Using Equation (44), it is directly verified that expression (40) is the explicit solution of Equation (37) if $\delta=\alpha-\gamma(\alpha)>n-1$, where $n-1=[\alpha]$ for non-integer values of $\alpha>0$. As a result, the condition $\alpha-\gamma(\alpha)>n-1$ (or $(\alpha+\beta) /(a-1)>n-1)$ with $t>0$ should be satisfied for parameters of Equation (40) with the Caputo derivative instead of $\alpha-\gamma(\alpha) \geq 0$ that is used in [29].

Remark 2. Note that in equations 3.5.48 and 3.5 .49 of [29] p. 209, there are typos: The signs of the parameters $\gamma(\alpha)$ and $\alpha$ in the gamma functions must be opposite. In Equation (40), these signs are correct.

Taking into account these Remarks and Propositions 3.8 and 3.9 of [29] pp. 209-210, we can formulate the following conditions for the existence of solution (40) for Equation (37).

Statement 1. Non-linear fractional differential Equation (37) with $\alpha \in(n-1, n), n \in \mathbb{N}$, has the solution, which is given by Equations (40) and (41), if the following conditions are satisfied:

$$
\left\{\begin{array} { c } 
{ a \in ( 0 , 1 ) , } \\
{ \beta > - \alpha - ( n - 1 ) ( a - 1 ) , }
\end{array} \quad \text { or } \left\{\begin{array}{c}
a>1, \\
\beta<-\alpha-(n-1)(a-1) .
\end{array}\right.\right.
$$

Here, we take into account that $\delta=\alpha-\gamma(\alpha)>n-1$ instead of $\delta=\alpha-\gamma(\alpha)>0$ which is used in Propositions 3.8 and 3.9 of [29].

Conditions (46) for the parameters, under which solution (40) exists, are important for applications in economic models.

\subsection{Rate of Growth with Power-Law Memory}

For solution (40), the rate of growth with power-law memory at time $t>0$ is approximately equal to:

$$
R(\alpha)=\frac{\alpha-\gamma(\alpha)}{t}=\frac{\alpha+\beta}{(1-a) t^{\prime}}
$$

where $a \neq 1$. For case $\alpha=1$, which corresponds to the absence of memory, Equation (47) can be written in the following form:

$$
R(1)=\frac{1-\gamma(1)}{t}=\frac{1+\beta}{(1-a) t} .
$$

Let us consider the case when we have growth with memory, i.e., the increasing function $K(t)$ for non-integer values of $\alpha$. In this case, the growth rate with memory, $R(\alpha)$ should be positive for non-integer values of $\alpha \in(n-1, \alpha), n \in \mathbb{N}$, i.e., we have the following inequality:

$$
R(\alpha)>0 .
$$


Solutions (40) and (41) exist only if the following conditions are satisfied:

$$
\alpha-\gamma(\alpha)=\frac{\alpha+\beta}{1-a}>n-1, \gamma(\alpha)<1 .
$$

Obviously, using Equations (47) and (50), we get that condition (49) has the following form:

$$
R(\alpha)=\frac{\alpha-\gamma(\alpha)}{t}=\frac{\alpha+\beta}{(1-a) t}>0,
$$

which will be satisfied for any solutions for $t>0$. Mathematically, this statement follows from the fact that if $\alpha-\gamma(\alpha)>n-1$ with $n \in \mathbb{N}$, then $\alpha-\gamma(\alpha)>0$.

As a result, we can formulate the following principle.

Principle 1. Principle of Inevitability of Growth with Memory: For economic models with memory, which are described by Equations (5), (6), and (36) with $a \neq 1, q \neq 1$, and $p \neq 1$, the rate of growth with power-law memory (47) is positive.

$$
R(\alpha)=\frac{\alpha+\beta}{(1-a) t}=\frac{\alpha(1-p)(1-q)+\beta(2-p-q)}{(1-a)(1-p)(1-q) t}>0
$$

for non-integer values of $\alpha$. As a result, the inclusion of power-law memory effects leads to the inevitability of capital growth $K(t)$.

In addition, we have the lower boundary of the growth rates for processes with memory, which is described by the following statement.

Statement 2. The rate of growth with power-law memory for processes, which are described by Equation (37), has the lower boundary that is defined by the inequality:

$$
R(\alpha)>\frac{n-1}{t}
$$

where $n=[\alpha]+1$ for non-integer values of $\alpha$.

This statement is based on the fact that the solution exists only if the following condition holds:

$$
\alpha-\gamma(\alpha)=\frac{\alpha+\beta}{(1-a)}>n-1 .
$$

Using definition (47) of the rate of growth with memory, we get (53).

Remark 3. Solutions, in which the decline (recession) is realized, do not exist within the framework of the suggested model with memory. In this model, the processes with memory cannot have negative values of the rate of growth with a power-law memory. Note that for processes without memory, which are described by Equation (47) with $\alpha=1$, the negative growth rates $(R(1)<0)$ can be realized.

Let us consider the condition under which the growth rate with memory, $R(\alpha)$, is greater than the growth rate without memory, $R(1)$. This condition is represented by the inequality:

$$
R(\alpha)>R(1) .
$$

Using expressions (47) and (48), inequality (55) gives:

$$
R(\alpha)-R(1)=\frac{\alpha-1}{(1-a) t}>0,
$$


where we assume that $a \in(0,1)$.

Taking into account conditions (46) for the existence of solution (40) and that $n=1$ for $\alpha \in(0,1)$, we can formulate the following statement.

Statement 3. The rate $R(\alpha)$ of growth with memory for the capital $K(t)$ is greater than the rate $R(1)$ of growth without memory $R(\alpha)>R(1)$, if the following conditions are satisfied:

$$
\left\{\begin{array} { c } 
{ \alpha \in ( 0 , 1 ) , } \\
{ a > 1 , } \\
{ \beta < - \alpha , }
\end{array} \quad \text { or } \left\{\begin{array}{c}
\alpha \in(n-1, n), n \in \mathbb{N}, n \neq 1, \\
a \in(0,1), \\
\beta>-\alpha+(n-1)(1-a) .
\end{array}\right.\right.
$$

Let us consider the question of how much growth with memory can be greater than growth without memory. To describe this, we give the ratio of growth rates $R(\alpha)$ and $R(1)$. This ratio has the following form:

$$
\frac{R(\alpha)}{R(1)}=\frac{\alpha+\beta}{1+\beta},
$$

where we assume that $R(1)>0$, conditions (57) are satisfied, and $\beta$ is given by (39). Note that $\alpha \in(0,1)$, in inequality $R(\alpha)>R(1)$ is also satisfied since $a>1$ and $\alpha+\beta<1+\beta<0$.

\subsection{Dynamics of Capital Per Unit of Effective Labor}

The amount of capital per unit of effective labor [3] p. 10, is defined by the following equation:

$$
k(t)=\frac{K(t)}{A(t) L(t)} .
$$

The solution of capital in form (43) and expressions (12) and (14) allow us to describe the amount of capital per unit of effective labor (59) by the following equation:

$$
k(t)=\frac{K\left(t_{0}\right)}{A\left(t_{0}\right) L\left(t_{0}\right)}\left(\frac{t}{t_{0}}\right)^{\alpha-\gamma(\alpha)-1 /(1-q)-1 /(1-p)} .
$$

Remark 4. Note that the memory model described by the equation with a fractional derivative cannot be reduced to changing the parameterization of the standard model. This is due to the qualitatively different properties of the derivative of non-integer orders. For example, such standard properties of the first-order derivative, as the product rule and the chain rule, do not hold for fractional derivatives. For example, the expression $k(t)$, which is given by solutions of equations for $k(t)$, cannot coincide (for processes with memory) with the expression of $k(t)=K(t) / A(t) L(t)$, which is given by solutions of equations for $K(t)$, and $L(t), A(t)$. For standard models, both expressions are the same. We can state that the variable per capita (per unit of effective labor) constructed from the solutions of original variables is not a solution of the equation for the variable per capita (per unit of effective labor). Let us give a brief explanation of this statement (for a detailed description, see article [46]. Let us assume that we have the solution $L_{\text {sol }}(t)$ and $A_{\text {sol }}(t)$ of differential equations for $L(t)$ and $A(t)$, respectively. We can consider two variables $K(t)$ and $k(t)$ and two equations $K^{(1)}(t)=F(t, K(t))$ and $k^{(1)}(t)=f\left(t, k(t)\right.$. In the standard model, solutions $K_{\text {sol }}(t)$ and $k_{\text {sol }}(t)$ of these equations can be derived by the formula $K_{\text {sol }}(t)=k_{\text {sol }}(t) A_{\text {sol }}(t) L_{\text {sol }}(t)$. The fractional differential equations for the variables $K(t)$ and $k(t)$ cannot be related by such formulas, if the memory effect is taken into account.

For the capital per unit of effective labor $k(t)$, the rate of growth with power-law memory at $t>t_{0}$ is approximately equal to:

$$
r(\alpha)=\frac{\alpha-\gamma(\alpha)}{t}-\frac{1}{t}\left(\frac{1}{1-q}+\frac{1}{1-p}\right),
$$


where $q \neq 1, p \neq 1, a \neq 1$, and $\beta$ is defined by Equation (38). Expression (61) can be represented in the following form:

$$
r(\alpha)=\frac{\alpha+\beta}{(1-a) t}-\frac{\beta}{b t}=\frac{b(\alpha+\beta)-\beta(1-a)}{(1-a) b t} .
$$

For $\alpha=1$, which describes the case of absence memory, expression (62) takes the following form:

$$
r(1)=\frac{b(1+\beta)-\beta(1-a)}{(1-a) b t} .
$$

Expression (63) describes the rate of growth without memory for $a \neq 1$.

Let us consider the condition under which the growth rate with memory, $r(\alpha)$, is greater than the growth rate without memory, $r(1)$. This condition is represented by the following inequality:

$$
r(\alpha)>r(1) .
$$

Using Equations (62) and (63), inequality (64) takes the following form:

$$
r(\alpha)-r(1)=\frac{\alpha-1}{(1-a) t}>0 .
$$

Inequality (65) coincides with inequality (56). Therefore, Statement 3 and the system of inequalities (57) also should be satisfied for dynamics of the capital per unit of effective labor.

For the capital per unit of effective labor $k(t)$, the rate $r(\alpha)$ of growth with memory is greater than the rate $r(1)$ of growth without memory $r(\alpha)>r(1)$ if conditions (57) are satisfied.

Let us consider the question of how much growth with memory can be greater than growth without memory. Using Equations (62) and (63), the ratio of $r(\alpha)$ and $r(1)$ is expressed by the following equation:

$$
\frac{r(\alpha)}{r(1)}=\frac{\alpha b+\beta(a+b-1)}{b+\beta(a+b-1)}
$$

where we assume that $r(1)>0$, and the parameter $\beta$ is defined by Equation (38).

For $b=1-a$ with $a \in(0,1)$, we have the linearly homogeneous production functions Cobb-Douglas. In this case, the rate of growth with power-law memory (62) takes the following form:

$$
r(\alpha)=\frac{\alpha}{(1-a) t}
$$

Using that $\alpha \in(n-1, n)$, we get:

$$
r(\alpha)>\frac{n-1}{(1-a) t}
$$

for non-integer values of $\alpha$. In this case, the ratio of $r(\alpha)$ and $r(1)$ is expressed by the following equation:

$$
\frac{r(\alpha)}{r(1)}=\alpha
$$

Using that $\alpha \in(n-1, n)$, we obtain the following inequality:

$$
\frac{r(\alpha)}{r(1)}>n-1
$$

for non-integer values of $\alpha$.

Example 1. Using expression (69), we see that the rate of growth with memory can be several times (in $\alpha>1$ time) higher than the standard rate of growth without memory. For example, if $\alpha=3.2$, then the rate of growth 
with memory is $220 \%$ more (is 3.2 times more) than the standard rate of growth without memory, when other initial parameters of the models (with memory and without memory) are the same.

As a result, we find that the growth rate with memory is greater than the growth rate without memory by $\alpha$ times. This allows us to formulate the following principle, where we assume that conditions (57) are satisfied.

Principle 2. Principle of Changing Growth Rates by Memory: In the case $b=1-a$ with $a \in(0,1)$ and conditions (57) are satisfied, the rate of growth with a power-law memory for capital per unit effective labor can be greater than the rate of growth without memory $(r(\alpha)>r(1))$ in $\alpha$ times, if the parameter of memory fading $\alpha$ is more than one $(\alpha>1)$. The growth rate with memory is less than the growth rate without memory, i.e., $r(\alpha)<r(1)$, if the parameter $\alpha$ is less than one $(0<\alpha<1)$.

As a result, we can conclude that the fading memory can significantly (several times) change the growth rate, when other parameters of the model are unchanged. Therefore, we should not neglect the memory in economic models.

As a prospect for further studies of non-linear economic growth models with memory, it is possible to emphasize the need to take into account both simultaneous and sequential actions of the effects of memory and depreciation.

\section{Solow-Lucas Model of Closed Economy with Memory}

In this section, we consider a generalization of the standard growth model for closed economy without capital depreciation, which is considered by Robert E. Lucas in paper "Making a Miracle" [41]. In the generalized model, we take into account the memory with power-law fading. The suggested model with memory is described by the non-linear fractional differential equation. The explicit expression of analytical solution of this non-linear equation is obtained.

\subsection{Solow-Lucas Model for Closed Economy without Memory}

Let us consider the Solow model for closed economy that is described by Robert E. Lucas in [41] pp. 253-254 (see also Section 3.2 in [42] pp. 73-75). We will call this economic model the Solow-Lucas model. Note that Robert E. Lucas Jr received the Nobel Memorial Prize in Economic Sciences in 1995 "for having developed and applied the hypothesis of rational expectations, and thereby having transformed macroeconomic analysis and deepened our understanding of economic policy" $[43,44]$.

Let us consider an economy that uses physical capital $k(t)$ and the human capital $h(t)$ to produce a single good $y(t)$, the dynamics of which is described by the following equation:

$$
y(t)=A k^{a}(t)[u h(t)]^{1-a} .
$$

Here, the human capital input is multiplied by $u$, where $u$ is the fraction of time that people spend on producing goods.

The growth of human capital $h(t)$ depends on the amount of time spent on production, adjusted for quality (see Equation (13) in [45]) in the following form:

$$
\frac{d h(t)}{d t}=\delta(1-u) h(t)
$$

The solution of Equation (72) can be written as:

$$
h(t)=h(0) \exp \{\delta(1-u) t\},
$$

where $h(0)$ is the human capital at time $t=0$. 
The growth of physical capital depends on the savings rate $s$, such that we have the following equation:

$$
\frac{d k(t)}{d t}=s y(t)
$$

which is interpreted as an economic accelerator without memory and lag. The substitution of expressions (71) and (73) into Equation (74) gives:

$$
\frac{d k(t)}{d t}=s A u^{1-a} h^{1-a}(0) \exp \{\delta(1-a)(1-u) t\} k^{a}(t) .
$$

In this model, it is assumed that variables $s$ and $u$ are considered as given constants. The model, which is described by Equations (71)-(75), will turn out to be a Solow model rewritten for a closed economy [41,42].

In this model, the rate of technological changes (the average Solow balance) is described by the following expression:

$$
\lambda_{0}=\delta(1-a)(1-u),
$$

and the initial technology level is equal to $A h^{1-a}(0)$.

The long run growth rate of both capital and production per worker is $\delta(1-u)$. The growth rate of human capital, and the ratio of physical and human capital converge to constant values. In the long run, the level of income is proportional to the economy's initial stock of human capital.

Remark 5. Note that in the Solow-Lucas model, the average values of $k(t), h(t)$, and $y(t)$ are taken as original variables to build a model, in contrast to the Solow model that use $K(t), L(t), Y(t)$ as original variables. In standard models, the use of these two types of variables is equivalent. Unlike standard models, in models with memory the use of different types of variables leads to different models that are not equivalent in the general case [46]. This non-equivalence is associated with the violation of the standard form of the product rule for the fractional derivative of non-integer orders. As a consequence, we have a violation of the standard relationship of the rates of change of absolute (volume) and relative (specific) indicators.

\subsection{Solow-Lucas Model for Closed Economy with Memory}

In the proposed generalization of the standard Solow-Lucas model, we will use the generalizations of Equations (71), (72), and (74).

(1) We assume that the economy, which uses physical capital $k(t)$ and the human capital $h(t)$ to produce a single good $y(t)$, is described by the following equation:

$$
y(t)=A k^{a}(t)[u h(t)]^{b}
$$

where $u$ is the fraction of time people spend producing goods. If $b=1-a$, then Equation (77) gives Equation (71).

(2) The growth of human capital is assumed to be the power law in the following form:

$$
\frac{d h(t)}{d t}=\delta(1-u) h^{\theta}(t)
$$

If $\theta=1$, then Equation (78) gives Equation (72) of the standard model.

(3) Equation (74) does not take into account the memory effects, since the derivative $d k(t) / d t$ is determined by the behavior of $y(\tau)$ only at the same time of instant $\tau=t$, and does not take into 
account the history of changes of $y(\tau)$ in the past, when $\tau \in(0, t)$. To take into account the changes of $y(\tau)$ in the past, we can use the following equation:

$$
\frac{d k(t)}{d t}=\int_{0}^{t} s(t, \tau) y(\tau) d \tau
$$

where function $s(t, \tau)$ allows us to take into account that the growth of physical capital $k(t)$ depends on the changes of $y(\tau)$ in the past at $\tau \in(0, t)$. Function $s(t, \tau)$ can be interpreted as a memory function. In order to have standard dimensions of economic quantities, the time variable $t$ is considered as a dimensionless parameter, i.e., we changed the variable $t_{\text {old }} \rightarrow t_{\text {new }}=t_{\text {old }} / t_{c}$, where $t_{c}$ is a characteristic time of economic process.

An important property of memory is described by the principle of memory fading, which states that the increasing of the time interval leads to a decrease in the corresponding contribution $[7,33]$. Let us consider the power-law form of the memory fading. In this case, the memory function can be described by the following equation:

$$
s(t, \tau)=\frac{s}{\Gamma(\mu)}(t-\tau)^{\mu-1},
$$

where $s$ is the savings rate and $\Gamma(\alpha)$ is the gamma function. For function (80), Equation (79) can be written in the following form:

$$
\frac{d k(t)}{d t}=s\left(I_{R L ; 0+}^{\mu} y\right)(t),
$$

where $I_{R L ; 0+}^{\mu}$ is the Riemann-Liouville fractional integral of the order $\mu>0$, [29] pp. 69-70.

We should note that (81) with the Riemann-Liouville fractional integral can be considered as an approximation of the equations with generalized memory functions (79). In paper [47], using the generalized Taylor series in the Trujillo-Rivero-Bonilla form for the memory function, we proved that Equation (79) for a wide class of memory functions can be represented through the Riemann-Liouville fractional integrals (and the Caputo fractional derivatives) of non-integer orders.

The action of the Caputo derivative on Equation (81) gives:

$$
\left(D_{C ; 0+}^{\mu} k^{(1)}\right)(t)=s\left(D_{C ; 0+}^{\mu} I_{R L ; 0+}^{\mu} y\right)(t),
$$

where $D_{C ; 0+}^{\mu}$ is the Caputo fractional derivative of the order $\mu>0$, [29] pp. 90-99.

In Equation (82), we can use the property that the Caputo fractional derivative is the left inverse operator for the Riemann-Liouville fractional integral (see the equation of Lemma 2.21 in [29] p. 95), in the following form:

$$
\left(D_{C ; 0+}^{\mu} I_{R L ; 0+}^{\mu} y\right)(t)=y(t)
$$

for $y(t) \in L_{\infty}(0, t)$ or $y(t) \in C[0, t]$, and the property of the Caputo fractional derivative:

$$
\left(D_{C ; 0+}^{\mu} k^{(1)}\right)(t)=\left(D_{C ; 0+}^{\mu+1} k\right)(t) .
$$

Using expressions (83) and (84), Equation (82) can be written as:

$$
\left(D_{C ; 0+}^{\alpha} k\right)(t)=s y(t)
$$

with $\alpha=\mu+1$. If $\alpha=1$, then Equation (85) takes the form of (74).

The substitution of (77) into (85) gives the equation which describes the dynamics with memory for physical capital $k(t)$ in the following form:

$$
\left(D_{C ; 0+}^{\alpha} k\right)(t)=s A k^{a}(t)[u h(t)]^{b},
$$


where the behavior of $h(t)$ is given by Equation (78). Equation (86) describes the proposed generalization of the Solow-Lucas model for a closed economy with power-law memory.

For $\alpha=1$, Equation (86) describes the Solow-Lucas model without memory, where $b \neq 1-a$ and $\theta \neq 1$.

\subsection{Growth Rates of Closed Economy with Memory}

Let us obtain the solution of the non-linear fractional differential Equation (78) which describes the Solow-Lucas model of closed economy with power-law memory.

We first solve Equation (78) with $\theta \neq 1$ for the human capital. Equation (78) can be written as:

$$
\frac{d}{d t}\left(h^{1-\theta}(t)\right)=\delta(1-u)(1-\theta),
$$

Therefore, the solution of Equation (87) with $\theta \neq 1$ has the following form:

$$
h^{1-\theta}(t)=\delta(1-u)(1-\theta) t+c
$$

where we can use $c=h^{1-\theta}(0)$.

For simplicity, we will assume that $h(0)=0$ and $h\left(t_{0}\right)=1$. Then, we get $c=0$ and solution (88) can be written in the following form:

$$
h(t)=(\delta(1-u)(1-\theta))^{1 /(1-\theta)} t^{1 /(1-\theta)} .
$$

Equation (89) with $t=t_{0}>0$ has the following form:

$$
h\left(t_{0}\right)=(\delta(1-u)(1-\theta))^{1 /(1-\theta)} t_{0}^{1 /(1-\theta)},
$$

where $\delta(1-u)(1-\theta)>0$. Assuming that $h\left(t_{0}\right)=1$, we get:

$$
t_{0}=\frac{1}{\delta(1-u)(1-\theta)}
$$

and solution (89) can be represented by the following equation:

$$
h(t)=h\left(t_{0}\right)\left(\frac{t}{t_{0}}\right)^{1 /(1-\theta)} .
$$

The substitution of (92) into (86) gives:

$$
\left(D_{C ; 0+}^{\alpha} k\right)(t)=s A\left[u h\left(t_{0}\right)\right]^{b}\left(\frac{t}{t_{0}}\right)^{b /(1-\theta)} k^{a}(t),
$$

where $\alpha=\mu+1$ with $\mu>0$. Equation (93) can be written in the following form:

$$
\left(D_{C ; 0+}^{\alpha} k\right)(t)=\lambda t^{b /(1-\theta)} k^{a}(t),
$$

where $\theta \neq 1$ and the constant $\lambda$ is defined as:

$$
\lambda=s A\left[u h\left(t_{0}\right)\right]^{b} t_{0}^{-b /(1-\theta)} .
$$

Using equations 3.5.47 and 3.5.48 of Section 3.5.3 in [29] p. 209, the following equation:

$$
\left(D_{C ; 0+}^{\alpha} k\right)(t)=\lambda t^{\beta} k^{a}(t),
$$


with $\lambda \neq 0, a \neq 1, \alpha=\mu+1>1$, and

$$
\beta=\frac{b}{1-\theta}
$$

has the following solution:

$$
k(t)=\left(\frac{\Gamma(\alpha-\gamma(\alpha)+1)}{\lambda \Gamma(1-\gamma(\alpha))}\right)^{1 /(a-1)} t^{\alpha-\gamma(\alpha),}
$$

where $\lambda$ is defined by Equation (95), and

$$
\gamma(\alpha)=\frac{\beta+a \alpha}{a-1}, \alpha-\gamma(\alpha)=\frac{\beta+\alpha}{1-a} .
$$

The condition for the existence of a solution can be written as:

$$
\alpha-\gamma(\alpha)>n-1, \gamma(\alpha)<1 .
$$

As a result, the rate of growth with power-law memory $(\alpha \neq 1)$ at time $t>0$ is approximately equal to:

$$
R(\alpha)=\frac{\alpha-\gamma(\alpha)}{t}=\frac{\beta+\alpha}{(1-a) t}=\frac{\alpha+b-\alpha \theta}{(1-a)(1-\theta) t}
$$

for $t>t_{0}>0$ and $\theta \neq 1, a \neq 1$, where we use (97).

For $\alpha=1$, expression (101) takes the following form:

$$
R(1)=\frac{\beta+1}{1-a}=\frac{1+b-\theta}{(1-a)(1-\theta) t} .
$$

Expression (102) describes the rate of growth without memory for the case $\theta \neq 1, a \neq 1$.

Using that solution (98) exists if conditions (100) are satisfied, i.e., $\alpha-\gamma(\alpha)>n-1$, we get:

$$
R(\alpha)>\frac{n-1}{t}
$$

for non-integer values of $\alpha \in(n-1, n)$ and $t>0$.

As a result, we get that $R(\alpha)>0$ for non-integer values of $\alpha$. Therefore, the rate of growth with power-law memory is positive for $t>0$ and non-integer values of $\alpha$.

We should note that the generalized model, which is described by Equation (79) assumes that $\alpha=\mu+1$, where $\mu>0$. Therefore, we may not consider the case with $0<\alpha<1$.

Let us consider the condition, under which the rate of growth with memory, $R(\alpha)$, is greater than the rate of growth without memory, $R(1)$, that is:

$$
R(\alpha)>R(1) .
$$

Using expressions (101) and (102), inequality (104) gives:

$$
R(\alpha)-R(1)=\frac{\alpha-1}{(1-a) t}=\frac{\mu}{(1-a) t}>0,
$$

where we assume that $a \in(0,1)$.

Using Equation (105), we can state that for $a \in(0,1)$, we have $R(\alpha)>R(1)$ for all $t>0$. As a result, we can formulate the following principle.

Principle 3. Principle of Changing Growth Rates by Memory: If a solution of Equation (96) for the physical capital $k(t)$ exists and $a<1$, then the rate of growth with power-law memory, $R(\alpha)$, is greater than the rate of growth without memory, $R(1)$, i.e., $R(\alpha)>R(1)$. 
Using conditions (57) for the existence of solution (98), and that $\alpha=\mu+1$ with $\mu>0, m=n-1$, we can formulate the following statement.

Statement 4 . The rate $R(\alpha)$ of growth with memory for the physical capital $k(t)$ is greater than the rate $R(1)$ of growth without memory $(R(\alpha)>R(1))$, if the following conditions are satisfied:

$$
\left\{\begin{array}{c}
\mu \in(m-1, m) m \in \mathbb{N} \\
a \in(0,1) \\
\beta>-\mu-1+m(1-a)
\end{array} .\right.
$$

The ratio of $R(\alpha)$ and $R(1)$, which are given by expressions (101) and (102), is expressed by the following equation:

$$
\frac{R(\alpha)}{R(1)}=\frac{\alpha(1-\theta)+b}{1-\theta+b}=\frac{1-\theta+b+\mu(1-\theta)}{1-\theta+b} .
$$

For the special case $0<1-\theta=b<1$, Equation (107) gives:

$$
\frac{R(\alpha)}{R(1)}=\frac{\alpha+1}{2}=1+\frac{\mu}{2}
$$

where we use $\alpha=\mu+1$.

Example 2. Using expression (108), we see that the rate of growth with memory can be several times (in $\mu>0$ time) higher than the standard rate of growth without memory. For example, if $\alpha=3.2$ and $1-\theta=b \in(0,1)$, then the rate of growth with memory is $110 \%$ more (is 2.1 times more) than the standard rate of growth without memory, when other initial parameters of the models (with memory and without memory) are the same.

In this case, we see that the effects of memory can increase the growth rate by more than two times in comparison with the standard Solow-Lucas model without memory.

As a result, we can conclude that the memory effects can significantly (several times) change the growth rate, when other parameters of the model are unchanged, and we should not neglect the memory in economic models.

\section{Conclusions}

This article proposes generalizations of two well-known standard models with continuous time, which are described by Nobel laureates in economics, Robert M. Solow and Robert E. Lucas. In the suggested generalizations of the standard models, the memory with power-law fading is taken into account. As a mathematical tool for accounting for fading memory, the article uses integral derivatives of non-integer order. Note that the violation of the standard product (Leibniz) rule for fractional derivatives of non-integer orders leads to the non-equivalence of models that are equivalent in the absence of memory [46]. For the suggested model with memory, we derive non-linear fractional differential equations and its explicit analytical solutions. We prove that the rate of growth with power-law memory can be greater than the rate of growth without memory. For non-linear growth models with power-law memory, the growth rates can be changed by memory effects.

In this article, we proposed the following three principles, which can be used to describe the changes arising in the economic dynamics, when taking into account the power-law memory:

(I) The Principle of Inevitability of Growth with Memory states that in the first proposed model, which are described by Equations (5),(6) and (36), with $a \neq 1, q \neq 1$, and $p \neq 1$, the rate of growth with power-law memory (47) is positive (52) for non-integer values of $\alpha$. As a result, the inclusion of power-law memory effects leads to the inevitability of capital growth $K(t)$.

(II) The Principle of Changing Growth Rates by Memory states that in the first proposed model, the rate of growth with power-law memory for capital per unit of effective labor can be greater 
than the rate of growth without memory in $\alpha$ times, if the parameter of memory fading $\alpha$ is more than one $(\alpha>1)$.

(III) The Principle of Changing Growth Rates by Memory states that in the second proposed model, the rate of growth with power-law memory is greater than the rate of growth without memory, if a solution of Equation (96) for the physical capital $k(t)$ exists and $a<1$.

Therefore, we should emphasize that the memory effects can significantly (several times) change the growth rate, when other parameters of the model are unchanged, and we should not neglect the memory in economic models. For example, if $\alpha=3.2$, then the rate of growth with memory is $220 \%$ more ( 3.2 times more) and 110\% more ( 2.1 times more) for first and second proposed models, respectively than the standard rate of growth without memory.

Let us note here a possible direction for further mathematical research on non-linear models of economic growth with memory. An interesting and important part of future research is to account for both simultaneous and sequential actions of memory effects and depreciation. Standard economic models often use the exponential kernel of the integral operator to describe depreciation. In Equation (17), kernel $D(t-\tau)=e^{-\delta(t-\tau)}$ describes the standard (exponentially distributed) depreciation (up to a numerical factor) in form (18). The following types of integral operator kernels can be used to account for memory and depreciation effects in economic growth models:

(a) In Equation (17), kernel $D(t-\tau)=(t-\tau)^{\alpha-1} / \Gamma(\alpha)$ describes the interconnection of variables with power-law memory. In this case, integral operator (17) is called the Riemann-Liouville fractional integral. Economic models of this type were considered, for example, in works [7,33-36,38].

(b) We can use kernel $D(t-\tau)=e^{-\delta(t-\tau)}(t-\tau)^{\alpha-1} / \Gamma(\alpha)$, which describes the gamma distributed lag by Equation (17). The economic model with this type of distributed lag was considered in work [53]. Note that kernels of exponential amortization and power-law memory are special cases of this kernel for the case $\delta=0$ and $\alpha=1$, respectively.

(c) To describe the sequential action of two such effects as fading memory and continuously distributed delay (or continuously distributed amortization), it is necessary to use integral fractional operators with distributed lag, proposed in works [54] and then applying them in linear economic models [55-58].

(d) Kernel $D(t-\tau)=(t-\tau)^{\alpha-1} E_{\rho, \alpha}^{\gamma}\left[\omega(t-\tau)^{\rho}\right]$ describes the connection of variables with power-law memory and depreciation in special cases. Here, $E_{\rho, \alpha}^{\gamma}\left[\omega(t-\tau)^{\rho}\right]$ is the three-parameter Mittag-Leffler function [59,60], which is also called the Prabhakar function [61-63]. Integral operator (17) with this kernel is called the Prabhakar fractional integral [61-63]. The economic model with this type was considered in works [64,65], where a new economic model through learning-by-doing with memory was proposed. For the first time, the fractional derivative of Riemann-Liouville type, which has the Prabhakar function in the kernel, was proposed by Anatoly A. Kilbas, Megumi Saigo, and Ram K. Saxena in work [61] in 2004. This operator can be known as the Kilbas-Saigo-Saxena (KSS) fractional derivative. Note that the KSS operators are left-inverse for the Prabhakar fractional integrals [61]. Then, the fractional derivatives and integrals began to be studied in various papers (for example, see [62-64] and references therein). An application of these operators in economics with memory is proposed in papers $[64,65]$. We should emphasize that the main property of any generalized (fractional) derivative is to be a left-inverse operator to the corresponding generalized (fractional) integral operator. This requirement is important for a self-consistent mathematical theory of the fractional operators to have a general fractional calculus of these operators.

Funding: This research received no external funding.

Conflicts of Interest: The author declares no conflict of interest. The funders had no role in the design of the study; in the collection, analyses, or interpretation of data; in the writing of the manuscript, or in the decision to publish the results. 


\section{References}

1. Solow, R.M. A contribution to the theory of economic growth. Q. J. Econ. 1956, 70, 65-94. Available online: http://piketty.pse.ens.fr/files/Solow1956.pdf (accessed on 12 October 2020). [CrossRef]

2. Solow, R.M. Neoclassical growth theory. In Handbook of Macroeconomics; Elsevier Science B.V.: Amsterdam, The Netherlands, 1999; Volume 1, pp. 637-667. [CrossRef]

3. Romer, D. Advanced Macroeconomics, 3rd ed.; McGraw-Hill Companies: Boston, MA, USA, 2006; 678p, ISBN 978-0-07-287730-8.

4. Barro, R.J.; Sala-i-Martin, X.I. Economic Growth, 2nd ed.; The MIT Press: Cambridge, MA, USA; London, UK, 2003; p. 654. ISBN 978-0262025539.

5. Volgina, O.A.; Golodnaya, N.Y.; Odiyako, N.N.; Shuman, G.I. Mathematical Modeling of Economic Processes and Systems, 3rd ed.; Knorus: Moscow, Russia, 2016; p. 196. ISBN 978-5-406-04805-4.

6. NobelPrize.org. The Sveriges Riksbank Prize in Economic Sciences in Memory of Alfred Nobel 1987. Press Release. Nobel Media AB 2019. Available online: https://www.nobelprize.org/prizes/economicsciences/1987/press-release/ (accessed on 12 October 2020).

7. Tarasova, V.V.; Tarasov, V.E. Concept of dynamic memory in economics. Commun. Nonlinear Sci. Numer. Simul. 2018, 55, 127-145. [CrossRef]

8. Granger, C.W.J. The Typical Spectral Shape of an Economic Variable; Technical Report; Department of Statistics, Stanford University: Stanford, CA, USA, 1964; Volume 11, pp. 1-21. Available online: https://statistics. stanford.edu/research/typical-spectral-shape-economic-variable (accessed on 12 October 2020).

9. Granger, C.W.J. The typical spectral shape of an economic variable. Econometrica 1966, 34, 150-161. [CrossRef]

10. Granger, C.W.J. Essays in Econometrics: Collected Papers of Clive W.J. Granger. In Spectral Analysis, Seasonality, Nonlinearity, Methodology, and Forecasting; Ghysels, E., Swanson, N.R., Watson, M.W., Eds.; Cambridge University Press: Cambridge, UK; New York, NY, USA, 2001; Volume I, p. 523.

11. Granger, C.W.J.; Joyeux, R. An introduction to long memory time series models and fractional differencing. J. Time Ser. Anal. 1980, 1, 15-39. [CrossRef]

12. Granger, C.W.J. Essays in Econometrics Collected Papers of Clive W.J. Granger. In Causality, Integration and Cointegration, and Long Memory; Ghysels, E., Swanson, N.R., Watson, M.W., Eds.; Cambridge University Press: Cambridge, UK, 2001; Volume II, p. 398. ISBN 978-0-521-79207-3.

13. NobelPrize.org. The Sveriges Riksbank Prize in Economic Sciences in Memory of Alfred Nobel 2003. Press Release. Nobel Media AB 2019. Available online: https://www.nobelprize.org/prizes/economicsciences/2003/summary/ (accessed on 12 October 2020).

14. Beran, J. Statistics for Long-Memory Processes; Capman and Hall: New York, NY, USA, 1994; 315p, ISBN 0-412-04901-5.

15. Beran, J.; Feng, Y.; Ghosh, S.; Kulik, R. Long-Memory Processes: Probabilistic Properties and Statistical Methods; Springer: Berlin/Heidelberg, Germany; New York, NY, USA, 2013; 884p, ISBN 978-3-642-35511-0. [CrossRef]

16. Palma, W. Long-Memory Time Series: Theory and Methods; Wiley-InterScience: Hoboken, NJ, USA, 2007; 304p, ISBN 978-0-470-11402-5. [CrossRef]

17. Robinson, P.M. Time Series with Long Memory; Series: Advanced Texts in Econometrics; Oxford University Press: Oxford, UK, 2003; 392p, ISBN 978-0199257300.

18. Teyssiere, G.; Kirman, A.P. Long Memory in Economics; Springer: Berlin/Heidelberg, Germany, 2007; 389p. [CrossRef]

19. Tschernig, R. Wechselkurse, Unsicherheit und Long Memory; Physica-Verlag: Heidelberg, Germany, 1994; 232p, ISBN 978-3-7908-0753-0. [CrossRef]

20. Granger, C.W.J. Current perspectives on long memory processes. Acad. Econ. Pap. 2000, 28, 1-16. [CrossRef]

21. Baillie, R.N. Long memory processes and fractional integration in econometrics. J. Econom. 1996, 73, 5-59. [CrossRef]

22. Parke, W.R. What is fractional integration? Rev. Econ. Stat. 1999, 81, 632-638. [CrossRef]

23. Banerjee, A.; Urga, G. Modelling structural breaks, long memory and stock market volatility: An overview. J. Econom. 2005, 129, 1-34. [CrossRef]

24. Gil-Alana, L.A.; Hualde, J. Fractional Integration and Cointegration: An Overview and an Empirical Application. In Palgrave Handbook of Econometrics. Volume 2: Applied Econometrics; Mills, T.C., Patterson, K., Eds.; Springer: Berlin, Germany, 2009; pp. 434-469. [CrossRef] 
25. Grunwald, A.K. About "limited" derivations their application. J. Appl. Math. Phys. 1867, 12, 441-480.

26. Letnikov, A.V. Theory of Differentiation with Arbitrary Pointer. Mat. Sb. 1868, 3, 1-68. Available online: http://mi.mathnet.ru/eng/msb8039 (accessed on 12 October 2020).

27. Samko, S.G.; Kilbas, A.A.; Marichev, O.I. Fractional Integrals and Derivatives Theory and Applications; Gordon and Breach: New York, NY, USA, 1993; 1006p, ISBN 9782881248641.

28. Podlubny, I. Fractional Differential Equations; Academic Press: San Diego, CA, USA, 1998; p. 340.

29. Kilbas, A.A.; Srivastava, H.M.; Trujillo, J.J. Theory and Applications of Fractional Differential Equations; Elsevier: Amsterdam, The Netherlands, 2006; 540p.

30. Diethelm, K. The Analysis of Fractional Differential Equations: An Application-Oriented Exposition Using Differential Operators of Caputo Type; Springer: Berlin, Germany, 2010; 247p. [CrossRef]

31. Kochubei, A.; Luchko, Y. Handbook of Fractional Calculus with Application. Volume 1. Basic Theory. Walter de Gruyter GmbH: Berlin, Germany; Boston, MA, USA, 2019; 481p, ISBN 978-3-11-057081-6. [CrossRef]

32. Kochubei, A.; Luchko, Y. Handbook of Fractional Calculus with Applications. Volume 2. Fractional Differential Equations; Walter de Gruyter GmbH: Berlin, Germany; Boston, MA, USA, 2019; 519p, ISBN 978-3-11-057082-3. [CrossRef]

33. Tarasov, V.E.; Tarasova, V.V. Economic Dynamics with Memory: Fractional Calculus Approach; Walter de Gruyter GmbH: Boston, MA, USA, 2021; 678p, ISBN 978-3-11-062460-1.

34. Tarasova, V.V.; Tarasov, V.E. Macroeconomic models with dynamic memory: Fractional calculus approach. Appl. Math. Comput. 2018, 338, 466-486. [CrossRef]

35. Tarasov, V.E. Economic models with power-law memory. In Handbook of Fractional Calculus with Applications: Volume 8. Applications in Engineering, Life and Social Sciences, Part B; Baleanu, D., Lopes, A.M., Machado, J.A.T., Eds.; De Gruyter: Berlin, Germany; Boston, MA, USA, 2019; Chapter 1; pp. 1-32. ISBN 978-3-11-057092. [CrossRef]

36. Tarasov, V.E. Fractional econophysics: Market price dynamics with memory effects. Phys. A Stat. Mech. Appl. 2020, 557, 124865. [CrossRef]

37. Tarasova, V.V.; Tarasov, V.E. Dynamic intersectoral models with memory that generalize Leontief model. J. Econ. Entrep. 2017, 2, 913-924. Available online: https://elibrary.ru/item.asp?id=28791089 (accessed on 12 October 2020).

38. Tarasova, V.V.; Tarasov, V.E. Dynamic intersectoral models with power-law memory. Commun. Nonlinear Sci. Numer. Simul. 2018, 54, 100-117. [CrossRef]

39. Tarasov, V.E. On history of mathematical economics: Application of fractional calculus. Mathematics 2019, 7, 509. [CrossRef]

40. Tarasov, V.E. Mathematical Economics: Application of Fractional Calculus; MDPI: Basel, Switzerland; Beijing, China, 2020; 278p, ISBN1 978-3-03936-118-2. ISBN2 978-3-03936-119-9. [CrossRef]

41. Lucas, R.E. Making a Miracle. Econometrica 1993, 61, 251-272. Available online: http://www.dklevine.com/ archive/refs42101.pdf (accessed on 12 October 2020). [CrossRef]

42. Lucas, R.E. Lectures on Economic Growth; Harvard University Press: Cambridge, UK; London, UK, 2002; 204p, ISBN 0-674-00627-5.

43. NobelPrize.org. The Sveriges Riksbank Prize in Economic Sciences in Memory of Alfred Nobel 1995. Nobel Media AB 2019. Available online: https:/www.nobelprize.org/prizes/economic-sciences/1995/ summary/ (accessed on 12 October 2020).

44. The Royal Swedish Academy of Sciences. The Scientific Contributions of Robert E. Lucas, Jr. 1995. Available online: https:/www.nobelprize.org/prizes/economic-sciences/1995/advanced-information/ (accessed on 12 October 2020).

45. Lucas, R.E. On the mechanics of economic development. J. Monet. Econ. 1988, 22, 3-42. [CrossRef]

46. Tarasov, V.E. Rules for fractional-dynamic generalizations: Difficulties of constructing fractional dynamic models. Mathematics 2019, 7, 554. [CrossRef]

47. Tarasov, V.E. Generalized memory: Fractional calculus approach. Fractal Fract. 2018, 2, 23. [CrossRef]

48. Hilfer, R.; Luchko, Y. Desiderata for fractional derivatives and integrals. Mathematics 2019, 7, 149. [CrossRef]

49. Moiseev, N.N. Simplest Mathematical Models of Economic Forecasting; Znanie: Moscow, Russia, 1975; 64p, Available online: https://booksee.org/book/505314 (accessed on 12 October 2020).

50. Ivanilov, Y.P.; Lotov, A.V. Mathematical Models in Economics; Nauka: Moscow, Russia, 1979; 304p, Available online: http://www.library.fa.ru/files/Ivanilov.pdf (accessed on 12 October 2020).

51. Lotov, A.V. Introduction to Economic and Mathematical Modeling; Nauka: Moscow, Russia, 1984; 392p.

52. Allen, R.G.D. Mathematical Economics, 2nd ed.; Macmillan: London, UK, 1963; 812p, ISBN 978-1-349-81547-0. [CrossRef] 
53. Tarasov, V.E.; Tarasova, V.V. Logistic equation with continuously distributed lag and application in economics. Nonlinear Dyn. 2019, 97, 1313-1328. [CrossRef]

54. Tarasov, V.E.; Tarasova, S.S. Fractional and integer derivatives with continuously distributed lag. Commun. Nonlinear Sci. Numer. Simul. 2019, 70, 125-169. [CrossRef]

55. Tarasov, V.E.; Tarasova, V.V. Harrod-Domar growth model with memory and distributed lag. Axioms 2019, 8, 9. [CrossRef]

56. Tarasov, V.E.; Tarasova, V.V. Phillips model with exponentially distributed lag and power-law memory. Comput. Appl. Math. 2019, 38, 13. [CrossRef]

57. Tarasov, V.E.; Tarasova, V.V. Dynamic Keynesian model of economic growth with memory and lag. Mathematics 2019, 7, 178. [CrossRef]

58. Tarasov, V.E.; Tarasova, V.V. Dynamic Keynesian model of economic growth with memory and lag. In Advanced Mathematical Methods Theory and Applications; Mainardi, F., Giusti, A., Eds.; MDPI: Basel, Switzerland; Beijing, China; Wuhan, China; Barcelona, Spain; Belgrade, Serbia, 2020; 198p, pp. 116-132. ISBN 978-3-03928-247-0. [CrossRef]

59. Gorenflo, R.; Kilbas, A.A.; Mainardi, F.; Rogosin, S.V. Mittag-Leffler Functions, Related Topics and Applications; Springer: Berlin, Germany, 2014; p. 443. [CrossRef]

60. Prabhakar, T.R. A Singular Integral Equation with a Generalized Mittag-Leffler Function in the Kernel. Yokohama Math. J. 1971, 19, 7-15. Available online: https://irdb.nii.ac.jp/00822/0001826467 (accessed on 12 October 2020).

61. Kilbas, A.A.; Saigo, M.; Saxena, R.K. Generalized Mittag-Leffler function and generalized fractional calculus operators. Integral Transform. Spec. Funct. 2004, 15, 31-49. [CrossRef]

62. Giusti, A.; Colombaro, I.; Garra, R.; Garrappa, R.; Polito, F.; Popolizio, M.; Mainardi, F. A practical guide to Prabhakar fractional calculus. Fract. Calc. Appl. Anal. 2020, 23, 9-54. [CrossRef]

63. Giusti, A. General fractional calculus and Prabhakar's theory. Commun. Nonlinear Sci. Numer. Simul. 2020, 83, 105114. [CrossRef]

64. Tarasov, V.E.; Tarasova, S.S. Fractional derivatives and integrals: What are they needed for? Mathematics 2020, 8, 164. [CrossRef]

65. Tarasov, V.E. Fractional nonlinear dynamics of learning with memory. Nonlinear Dyn. 2020, 100, 1231-1242. [CrossRef]

Publisher's Note: MDPI stays neutral with regard to jurisdictional claims in published maps and institutional affiliations.

(C) 2020 by the author. Licensee MDPI, Basel, Switzerland. This article is an open access article distributed under the terms and conditions of the Creative Commons Attribution (CC BY) license (http://creativecommons.org/licenses/by/4.0/). 